\title{
Does artificial grass affect the competitive balance in major league soccer?
}

\author{
Matthew J. Trombley* \\ Abt Associates, Durham, NC, USA
}

\begin{abstract}
In this study I present cause for concern that Major League Soccer teams with an artificial grass (AG) home playing surface may possess an advantage over visiting teams used to playing on grass. I develop a theoretical model predicting the outcomes we should observe if teams with AG possess a competitive advantage. Using match-level data from the 2011-2014 Major League Soccer regular seasons, I find that conditional on attendance, distance traveled, and the difference in talent between the two teams, AG is uncorrelated with the final outcome of a match and the number of goals scored by each team. This suggests that AG does not affect the competitive balance in Major League Soccer.
\end{abstract}

Keywords: Association football, artificial turf, travel, attendance, match outcomes, restricted generalized Poisson, ordered logit

\section{Introduction}

Although soccer (association football) has traditionally been played on a natural grass surface, the game is increasingly played on artificial surfaces at all levels. In Major League Soccer (MLS), the highest level of professional soccer in the United States and Canada, four of the nineteen teams active in the 2014 season played their home matches on artificial grass (AG). Despite the increasing prevalence of AG at all levels, there remain many negative attitudes towards the surface among both players and coaches.

While serving as the US men's national team head coach, Bob Bradley called on FIFA (the international governing body of soccer) to ban the use of AG (Corr, 2009). Bruce Arena, Bradley's nationalteam predecessor (and current coach of the MLS' Los Angeles Galaxy) called the artificial surfaces in MLS “a disaster" (Davis, 2013). More recently, a group of high-profile female soccer players filed suit against both FIFA and the Canadian Soccer Association, protesting the use of AG for all matches at the 2015 Women's World Cup (Mccann, 2014).

There are at least three perceptions responsible for the common dislike of artificial grass. The first is

\footnotetext{
${ }^{*}$ Corresponding author: Matthew J. Trombley, Abt Associates, 5001S. Miami Blvd., Ste. 210, Durham, NC 27703, USA. Tel.: +1 919294 7846; E-mail: Matthew_Trombley@abtassoc.com.
}

the perception that $\mathrm{AG}$ increases the rate of injuries (Poulous et al., 2014). Although evidence is equivocal with regards to the probability of injury on real versus artificial grass, the effect on player behavior is real. Players in MLS, including marquee names such as David Beckham and Thierry Henry, have refused to play on AG, citing the increased probability of injury (Koreen, 2007; Pazino, 2013). The second perception players hold about AG is that it is more tiring to play on, and/or takes a greater physical toll in each game (Bell, 2009; Koreen, 2007; Gwynne, 2014; Davidson, 2014; Andersson, Ekblom, and Krustrup, 2008). Finally, the third perception is that the ball behaves differently on artificial versus real grass, moving faster and bouncing higher (Gwynne, 2014; Bell, 2009; Davidson, 2014; Andersson, Ekblom, and Krustrup, 2008). ${ }^{1}$

Each of these three perceptions may influence games played on AG rather than real grass, even if they are untrue. The most direct effect is if players opt to sit out games played on AG, presumably requiring

\footnotetext{
${ }^{1}$ The lawsuit against FIFA alleges that turf puts players at heightened risk of serious injury (perception 1), and 'fundamentally alters' the nature of the game being played (perception 3 and may encapsulate perception 2). Although the lawsuit is motivated primarily by issues of gender (since no men's World Cup match has ever been played on turf), a gender issue may not exist if the players did not feel so strongly that artificial surfaces are an inferior substitute for grass.
} 
the use of less-talented substitutes. There may also be indirect effects if players pace themselves differently on $\mathrm{AG}$, or if coaches implement more conservative game plans, due to the belief that they or their team will tire more quickly. ${ }^{2}$ Players and coaches on both teams may also change their behavior to avoid (or take advantage of) the possible miscues that may occur if the ball moves in unexpected ways.

While changing the nature of the game, however subtly, may affect the product on the field, if both teams are at an equal disadvantage, there should not be any effect on the competitive balance of the game. However, when star players from the visiting team opt to sit out matches played on AG, there is cause for concern that the home side obtains an advantage that would not have been gained on a natural surface. Moreover, it is likely that all of the other potential disadvantages of playing on AG fall more heavily on the visiting club (provided the visitors do not have an artificial home field). This is because players and coaches for these teams will likely have mentally adjusted to playing on turf, such that their behavior is no different on AG than grass. If AG truly alters the nature of the game, then the teams who play on AG regularly should have adjusted to the potential physical demands or changes in how the ball behaves more so than their opponents. Additionally, teams with AG are likely to be comprised of players who have self-selected to play on AG regularly, and thus these teams should not be deprived of their best players as frequently as visiting teams. ${ }^{3}$

To date, the literature regarding this subject has been limited. Barnett and Hilditch (1993) investigated the effect of AG on match results in the top four divisions of English soccer over the span from 1981 to 1989 . The authors find that teams with artificial playing surfaces earned 5-6 points more at home per season than would be predicted if the same team played on grass (equivalent to roughly two extra wins), and also had an improved home goal differential (the difference between goals scored and goals

\footnotetext{
${ }^{2}$ Andersson, Ekblom, and Krustrup (2008) fail to find any change in effort, headers, or standing tackles on artificial compared to real grass, but they do find that there are fewer slide tackles and more "short passes" on artificial compared to real grass.

${ }^{3}$ Teams with artificial surfaces also do not appear to have trouble landing top talent. Two of the most recent high-profile signings in MLS include US national-team standouts and World Cup veterans Clint Dempsey (signed with Seattle in 2013) and Jermaine Jones (signed with New England in 2014). However, it is possible that the physical toll of playing on AG regularly puts these teams at a potential disadvantage, something that will be revisited later.
}

conceded) of nearly 6 goals. Clarke and Norman (1995) examine the same data (extended through 1991) and find that teams with a grass home playing surface have a home advantage equivalent to 0.52 goals per match, while teams playing on AG enjoy a home advantage of 0.89 goals per game, a large and significant increase. However, AG has evolved considerably in the last 25 years, from plastic carpet overlaying concrete (think of a putt-putt playing surface) to advanced materials designed to closely emulate both grass and the underlying soil, and the literature has yet to revisit this issue.

This paper updates the investigation regarding the potential competitive influence of AG by analyzing match-level data from MLS spanning four seasons from 2011-2014. I consider whether teams with real grass home surfaces have a different probability of winning, drawing, and/or losing when playing matches on the road against opponents with AG rather than real grass. I also consider whether $A G$ affects the number of goals scored by the home team or the visiting team.

In addition to updating the literature to account for advances in AG technology, my study makes two substantive contributions to the literature. First, this study has an updated and expanded set of control variables compared to the previous literature. Unlike the prior two studies I control for differences in quality between the two opponents directly in the regression equation. I also control for travel distance and match attendance, two additional factors that have since been shown to be correlated with match outcomes.

Second, computing advances made in the past 20 years allow for the use of maximum likelihood methods that may fit the data better than traditional OLS methods, particularly count outcomes such as goals scored.

The conceptual model is discussed in Section 2 while Section 3 covers the selection of covariates and presents a brief descriptive analysis. Section 4 presents the empirical model and results. Section 5 provides several robustness checks for the results, and Section 6 concludes with a brief discussion of the findings.

\section{Conceptual model}

All possible outcomes of a soccer match are contained in the following equation:

$$
\mathrm{P}_{\mathrm{W}}+\mathrm{P}_{\mathrm{D}}+\mathrm{P}_{\mathrm{L}}=1
$$


where $\mathrm{P}_{\mathrm{W}}$ is the probability of winning, $\mathrm{P}_{\mathrm{D}}$ is the probability of a draw, and $\mathrm{P}_{\mathrm{L}}$ is the probability of a loss. These outcomes are mutually exclusive and exhaustive. Teams earn three points for a win, one point for a draw, and no points for a loss. In MLS, points determine who qualifies for the playoffs and the right to compete for the MLS championship. The team with the most points at the end of the season also earns the Supporters Shield, a trophy that crowns the champions of the regular season. Given these outcomes, teams seek to maximize expected points, where

$$
\mathrm{E}[\mathrm{P}]=3 \mathrm{P}_{\mathrm{W}}+1 \mathrm{P}_{\mathrm{D}}+0 \mathrm{P}_{\mathrm{L}} \cdot{ }^{4}
$$

In the following, I detail several assumptions about soccer matches, which lead to empirically testable predictions about the outcomes of interest.

Assumption 1: The three outcomes - win, draw, loss - are an ordered set that can be represented by the latent variable $\mathrm{Y}^{*}$, where the outcomes manifest as:

$$
\begin{aligned}
& \text { Loss }=\mathrm{Y}^{*}<\mu_{1} \\
& \text { Draw }=\mu_{1} \leq \mathrm{Y}^{*}<\mu_{2} \\
& \text { Win }=\mathrm{Y}^{*} \geq \mu_{2} .
\end{aligned}
$$

Assumption 2: Let $d$ be the relative (continuous) proportion of emphasis on defense versus offense selected by each team, where $d \in(0,1)$. Both teams maximize $E[P]$ by selecting $d^{*}$, taking into account all known information about the match, as well as the opposition's expected choice of $\mathrm{d}^{*}$. $\mathrm{d}^{*}$ is selected prior to the start of the match and is considered fixed.

This assumption states that the visiting team will adopt a point-maximizing balance of offense and defense based on what they know of the match itself and what they know of the opponent. So, for instance, if the visitor knows they have traveled particularly far for this match, making them more fatigued than usual, and that they are playing a team with a more dangerous attack than an average opponent, they will opt for a more conservative (i.e. defensive) strategy than they would have if they had not traveled or were playing a team with less emphasis on offense.

Assumption 3: Let E[GV] be the (continuous) number of goals expected for the visitor, and let $E\left[G_{H}\right]$

\footnotetext{
${ }^{4}$ In some cases, teams will not maximize expected points if they are in a situation where 1 point is as bad as 0 (e.g,., near the end of a season when only 3 points will keep a team in playoff contention). For simplicity, I assume that teams attempt to maximize points in all matches.
}

be the (continuous) number of goals expected for the home team. Then:

$$
\begin{aligned}
\frac{\partial \mathrm{E}\left[\mathrm{G}_{\mathrm{V}}\right]}{\mathrm{dAG}} \leq 0, \text { and } \\
\frac{\partial \mathrm{E}\left[\mathrm{G}_{\mathrm{H}}\right]}{\mathrm{dAG}} \geq 0 .{ }^{5}
\end{aligned}
$$

Assumption 3 states that AG does not have to disadvantage the visiting team, but if it does, it will do so by decreasing the number of goals scored by the visitors, and/or by increasing the number of goals scored by the home team.

Assumption 4: Let E[GD] be the (continuous) expected goal-differential between the two teams when both are playing a point maximizing strategy (in absence of $\mathrm{AG}$ ). If $\frac{\partial \mathrm{E}[\mathrm{GD}]}{\mathrm{dAG}}<0$ then $\frac{\partial \mathrm{E}\left[\mathrm{G}_{\mathrm{V}}\right]}{\partial \mathrm{d}_{V}}<$ $\frac{\partial \mathrm{E}\left[\mathrm{G}_{\mathrm{H}}\right]}{\partial \mathrm{d}_{V}}$ for some $d_{V}$, and therefore $d_{V}^{\prime}>d_{V}^{*}$, where $d_{V}^{\prime}$ is the visitor's level of defense adjusted for AG.

This assumption states that if the expected goal difference decreases from the perspective of the visitors (becomes less positive or more negative - i.e. the visiting team is disadvantaged by AG), then there is some level of additional defense such that the increase in defense will diminish the opponent's expected goals more than their own expected goals. This means it will be point maximizing behavior for the visitor to increase defense, and so the new level of defense $\left(d_{V}^{\prime}\right)$ will be greater than the point maximizing level of defense that would be observed in the absence of AG $\left(d_{V}^{*}\right)$.

Assumption 5: If $\frac{\partial \mathrm{E}[\mathrm{GD}]}{\mathrm{dAG}}<0$ then $\frac{\partial d_{H}}{d A G}+\frac{\partial d_{H}}{\partial \mathrm{d}_{V}} \leq 0$.

This assumption states that if $A G$ disadvantages the visiting team, then the home team's total shift in defense, in response to both $\mathrm{AG}$ (i.e., $\left.\frac{\partial d_{H}}{\mathrm{dAG}}\right)$ and the visitor's anticipated defensive response to $A G$ (i.e., $\frac{\partial d_{H}}{\partial d_{V}}$ ), may result in no net change in strategy, or a decrease in defensive posture, but will never increase defensive posture.

Empirical Prediction 1: The number of goals scored by the visitor will decrease when playing on AG because even if AG does not directly decrease the visitor's goals, their shift towards a more defensive strategy will. However, the change in the number of goals scored by the home team on AG is ambiguous. AG may directly increase the number of goals scored by the home team. However, if AG disadvantages the visitor by diminishing the visitor's goals, and the

\footnotetext{
${ }^{5} \mathrm{AG}$ cannot have a partial derivative since it is a binary variable.
} 
visitor responds with increased defense, the home team may also see a reduction in expected goals.

\section{Assumption 6:}

$$
\begin{aligned}
& \left|\frac{\partial^{2} E\left[G_{V}\right]}{d A G \partial E\left[G_{V}\right]}\right|>0 \text { and } \\
& \left|\frac{\partial^{2} E\left[G_{H}\right]}{d A G \partial E\left[G_{H}\right]}\right|<0 .
\end{aligned}
$$

This assumption states that the more goals the visitor is expected to score, the greater the potential reduction in goals caused by AG. Conversely, the more goals the home team is expected to score, the smaller is the potential increase in goals caused by AG.

Assumption 7: Ceteris Paribus, the larger is $\mathrm{E}\left[\mathrm{G}_{\mathrm{V}}\right]$, and/or the smaller is $E\left[G_{H}\right]$, the greater is $P_{W}$ (and the smaller is $\mathrm{P}_{\mathrm{L}}$ ) for the visiting team.

This assumption states that if the visitor is expected to score many goals, and/or the home team is expected to score few, than the visitor will have a larger probability of winning and smaller probability of losing than if the opposite was true (and vice versa).

Assumption 8: By Assumptions 6 and 7, I assert that:

$$
\frac{\partial P_{D}}{\partial P_{W}}>\frac{\partial P_{D}}{\partial P_{L}} .
$$

This assumption states that it is easier for a win to become a draw than for a draw to become a loss. This follows from assumptions 6 and 7. If the visitors are expected to win, it is because they have more expected goals than the home team. On the other hand, if the visitors are expected to draw, it is because either a) they have lower expected goals than if they were expected to win and/or b) the home team has more expected goals than if the visitor was expected to win. This means that the potential for reduced goals by the visitor due to $\mathrm{AG}$, or increased goals by the home team due to $\mathrm{AG}$, is higher if the visitor is expected to win (absent AG) than if the visitor is expected to draw. If the visitor is more likely to encounter a disadvantage from an initial position of victory than stalemate, it follows that, on the margin, it is easier for AG to turn a win into a draw than a draw to a loss.

Empirical Prediction 2: $A G$ will decrease $\mathrm{P}_{\mathrm{W}}$ for the visitor since either a reduction in their own expected goals, or an increase in the home team's expected goals, will decrease their probability of winning. Similarly, $A G$ will increase $P_{L}$ since a reduction in the visitor's expected goals or an increase in the home team's expected goals will also increase the probability of losing. Lastly, AG is predicted to increase $\mathrm{P}_{\mathrm{D}}$ since, on the margin, more wins will be turned into draws than draws turned into losses.

\section{Descriptive analysis and variables}

Following Barnett and Hilditch (1993) I begin with a descriptive analysis comparing average results at home and away for teams with grass home playing surfaces and those with an AG home surface (Table 1). I consider points earned per game, the probability of win, draw, or loss, goals scored, and goals conceded. Columns 2-5 show results earned at home and away separately for teams with grass and AG home playing surfaces (i.e., column 4 of Table 1 shows averages for teams with grass home playing surfaces for all matches they played away from home, regardless of whether or not the match itself was played on grass or AG).

If $\mathrm{AG}$ yielded an additional home advantage, we would expect the difference between results at home and results away to be larger for teams with AG.

Table 1

Average match outcomes by home playing surface

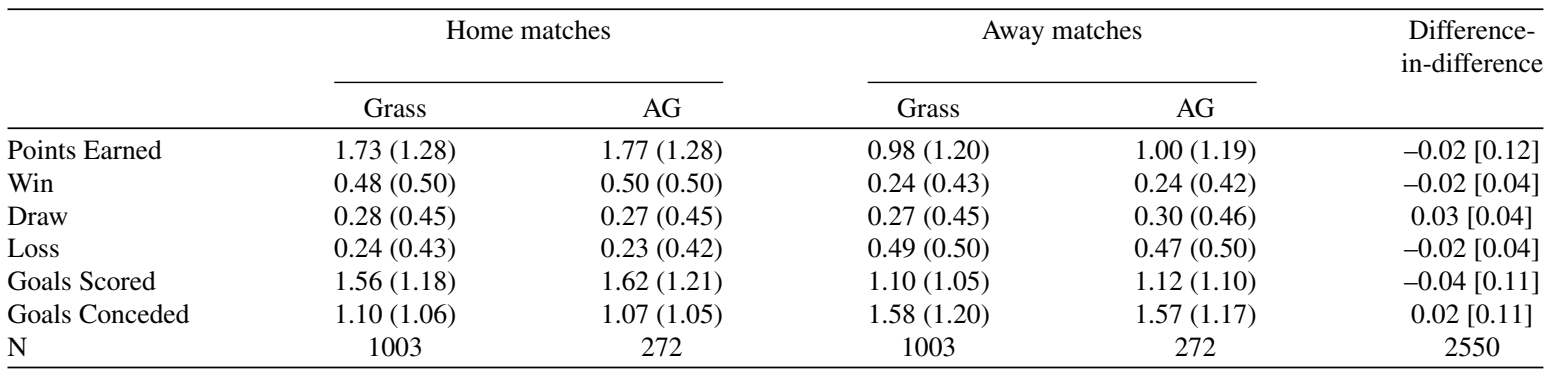

Statistical significance determined by $t$-test. Due to rounding, column 6 may not exactly equal the difference-in-difference between columns 2-5. Standard deviations of means in parentheses. Standard error of difference-in-difference estimates in brackets. 
Therefore, for each outcome I use OLS to compute an unconditional difference-in-difference estimate that shows whether the difference between results at home and results away differs based on whether a team has a natural home surface or AG. This estimate is reported in column 6 of Table 1 .

I find no significant difference between teams with AG and teams with natural grass for results at home or results away. Additionally, the differencein-difference estimate is small and insignificant for all results, suggesting that the gap between home and away performance is uncorrelated with AG.

However, estimating the effect of AG on match outcomes and goals scored is complicated by the fact that AG may not be independent of all factors correlated with the outcome of a game. There are several factors identified in the literature as correlated with the outcomes of competitive games that may be spuriously correlated with the probability of having a natural grass field, and which will bias the estimated effect of AG if not accounted for.

The most substantial predictor of a match outcome is likely the discrepancy in talent or skill between the two teams. Talent may be correlated with the probability of having an AG playing surface for two reasons. The first is that, with the exception of the New England Revolution, three of the four teams in MLS with artificial surfaces are recent expansions teams (i.e., new members of the league) relative to the data. ${ }^{6}$ In general, expansion teams begin with below-average performance. ${ }^{7}$ The second is that performance in MLS, a league that emphasizes parity, is fairly noisy from year to year, and so it is possible that the four years under observation are spuriously correlated with especially weak or strong seasons for the teams with artificial playing surfaces.

A common approach to approximating the difference in skill between two soccer teams is to use the difference in total points earned, or the difference in "win ratio" where each win is worth 1 , each draw is worth 0.5 , and a loss is worth 0 (Ponzo and Scoppa, 2014; Arabzad et al., 2014; Chumacero, 2007; Goddard, 2005). Summations of points or win ratios are

\footnotetext{
${ }^{6}$ Seattle began playing in MLS in 2009, while Vancouver and Portland both joined in 2011 (the first year of my data).

${ }^{7}$ In 1998 expansion side Tampa Bay Mutiny finished 9th out of the then 12 teams. In 2005, Chivas USA and the Houston Dynamo finished in 11th and 12th place out of 12. Toronto FC finished 13th of 13 in 2007. In 2010, the Philadelphia Union finished 14th out of 16. In 2012 the Montreal Impact finished 12th of 19. One notable exception is the 1998 Chicago Fire who finished 3rd in the league, and went on to win MLS Cup that year. (Major League Soccer, 2014)
}

computed for either the entirety of the season, or the most recent set of games. I measure skill using total points rather than win ratio, and sum points earned over the entire season rather than the most recent games. $^{8}$

One complicating factor for the analysis is that points earned at home by teams with AG may be biased upward if they possess a competitive advantage at home. Additionally, points earned away from home by teams that play on grass surfaces may be downward biased if many of their away games are against teams with AG. To account for this, I use the difference between total points earned at home (for the visiting team) and total points earned on the road (for the home team). This prevents the type of contamination discussed above while preserving the relative differences in skill between teams. ${ }^{9}$ Due to this approach, I limit the analysis to matches played on the road by teams with grass home playing fields.

The literature also suggests that travel distance influences the outcome of soccer matches due to an increase in player fatigue (Oberhofer, Philippovich, and Winner, 2010; Clarke and Norman, 1995, Goumas, 2014b). Since the four teams with artificial surfaces are located on one of the two coasts, and fairly high north, it is possible that teams may have to travel above-average distances to play against these teams. To control for this, I include a measure of miles traveled by the visiting team (DistanceFromTo, 2014). ${ }^{10}$

Evidence also indicates that the crossing of time zones conditional on distance (and the resultant potential for jet lag) is another important predic-

\footnotetext{
${ }^{8}$ I estimated models using win ratio, and also using points earned from the most recent four games (four being a number selected by both Ponzo and Scoppa [2014] and Arabzad et al., [2014].) Neither variable specification made a substantive difference in model fit or in the estimated marginal effect of AG for match outcomes, goals scored, or goals conceded. Results of these additional specifications are reported in Appendix C.

${ }^{9}$ Unfortunately, the data do not support the assumption that points(away) is a monotonic transformation of points(home) (i.e., the rank order of teams by home points is not the same as the rank order by away points). However, this simply means that the difference in skill between the two teams is measured with error. So long as the measurement error is not a function of the outcome of that particular game, nor of the probability of having an artificial playing surface, the measurement error should not bias estimates of the effect of AG. This assumption will be revisited in Section 5 .

${ }^{10}$ This refers to flight-distance between cities, not driving distance, nor distance from stadium to stadium. I also cannot control for the mode of travel, although it is possible that teams that are geographically close together may take busses or trains between games. However, this shortcoming is shared by the rest of the literature.
} 
tor of match results in both American football and Australian soccer (Goumas, 2014a; Nichols, 2012). Teams playing against clubs with AG may have to cross up to three time zones (whereas many teams can be visited with a maximum of two changes in time zone) and so AG may be correlated with increased jet lag. As Nichols (2012) points out, traveling east and west may have different short-term physiological effects, and so I include separate dummy variables for travel of 1,2, or 3 time zones west, and 1, 2, and 3 time zones east.

The literature has also found some (albeit limited) evidence that attendance influences the outcome of matches in favor of the home team (Ponzo and Scoppa, 2014), although Dewenter and Namini (2013) find that the home crowd may actually encourage home teams to deviate from a point-optimizing strategy. Three of the teams (Vancouver, Portland, Seattle) with AG have higher than average attendance, while the other (New England) has below average. ${ }^{11}$ Therefore, there may be unobserved correlation between match outcomes and AG. To control for the effect of the home crowd I include a measure of the mean average attendance for the home team in the year the match was played, as well as a squared attendance term.

Lastly, to round out the linear indices, I include fixed effects for the visiting team (the subject of the outcome estimate) as well as fixed effects for the year in which the match was played. ${ }^{12} \mathrm{I}$ argue that conditional on all of these variables the model is comprehensive and will produce an unbiased estimate of the effect of artificial playing surfaces on the outcome of the match, as well as the number of goals scored by each team. All match outcomes, point totals, goals for the home and visiting team, and average attendance figures are obtained from the official MLS website (Major League Soccer, 2014)

Table 2 compares mean outcomes for away matches played on grass versus those played on AG by teams with home grass surfaces. Additionally Table 2 compares average values for each of the variables hypothesized to confound the estimates of AG, including difference in points earned, distance traveled,

\footnotetext{
${ }^{11}$ Seattle led the league in mean attendance all four years. Portland was in the top five every year, Vancouver was in the top 5 three out of four years (and ranked 6 the other year), and New England was in the bottom five in attendance every year (Major League Soccer, 2014).

${ }^{12}$ Each team has 17 away matches per year. Research by Heckman (1981) has indicated that 8 observations is sufficient to avoid the problem of incidental parameter bias in binary models, and Allison and Waterman (2002) contends that this holds for count models as well.
}

Table 2

Average values of dependent and independent variables by opponent's playing surface

\begin{tabular}{lcc}
\hline & $\begin{array}{c}\text { Opponent has } \\
\text { Grass Surface }\end{array}$ & $\begin{array}{c}\text { Opponent has } \\
\text { AG Surface }\end{array}$ \\
\hline Dependent Variables & & \\
Probability of a Win & $24.04 \%(42.76)$ & $22.54 \%(41.86)$ \\
Probability of a Draw & $27.47 \%(44.67)$ & $26.18 \%(44.04)$ \\
Probability of a Loss & $48.49 \%(50.00)$ & $51.27 \%(50.07)$ \\
Goals Scored By & $1.11(1.06)$ & $1.07(1.02)$ \\
Visiting Team & & \\
Goals Conceded By & $1.57(1.17)$ & $1.63(1.26)$ \\
Visiting Team & & \\
Independent Variables & & $12.36(10.73)$ \\
Difference in Points & $12.62(9.22)$ & $22230.84 *$ \\
Opponent Attendance & 17384.24 & $(10635.25)$ \\
& $(3251.47)$ & $1322.00^{*}(710.89)$ \\
Distance & $978.16(631.10)$ & $31.64 \% *(46.59)$ \\
No time zone crossed & $23.49 \%(42.42)$ & $14.18 \%^{*}(34.95)$ \\
1 time zone East & $34.48 \%(47.56)$ & $1.09 \% *(10.41)$ \\
2 time zones East & $6.04 \%(23.85)$ & $6.18 \%(24.13)$ \\
3 time zones East & $7.55 \%(26.45)$ & $14.18 \%(34.95)$ \\
1 time zones west & $12.09 \%(32.62)$ & $19.27 \% *(39.52)$ \\
2 time zones west & $14.29 \%(35.02)$ & $13.45 \% *(34.19)$ \\
3 time zones west & $2.06 \%(14.21)$ & $\mathrm{N}=275$ \\
& $\mathrm{~N}=728$ & \\
\hline A & & \\
\hline
\end{tabular}

AG refers to artificial grass. ${ }^{*} p<0.05$. Statistical significance determined by $t$-test. Standard deviations in parentheses.

match attendance, and time zones cross. It appears that none of the three outcomes of interest (match outcome, goals scored by the visiting team, and goals conceded by the visiting team) are significantly different for matches played on AG compared to grass. However, several of the independent variables are significantly different between AG and grass. Games played on AG are attended by nearly 5,000 additional fans, roughly $1 / 3$ greater than the number attending games played on grass. Teams traveling to play on AG must travel further than average, a difference of roughly 350 miles (again a difference of nearly 1/3). Although teams are significantly more likely to cross no time zones to play on AG, they are also less likely to cross time zones east and more likely to cross time zones westward. The potential confounding effect of these variables must be taken into account through rigorous regression analysis in order to isolate the true relationship between match outcomes and AG.

\section{Empirical models and results}

\subsection{Match outcomes}

Prior literature at the match level has frequently estimated winning, drawing, and losing, as an ordered outcome (see e.g., Ponzo and Scoppa, 2014; Graham and Stott, 2008; Goddard, 2005; Goddard and Asimakopoulos, 2004). Although such a specification 
maximizes the efficiency of the empirical model, it requires the potentially restrictive assumption of proportional odds, i.e., that the relationship between each of the three possible outcomes is the same. To test this assumption I estimate an ordered logit model and then run a Brant test (Brant, 1990) using the oparallel Stata user-written command developed by Buis (2013). The test reports a chi-squared statistic of 34.55 with 29 degrees of freedom. This fails to reject the null hypothesis of proportional odds $(p=0.220)$ indicating that the ordered logit model is appropriate for estimating match outcomes.

Another potential issue is the possibility that the latent variable $\left(\mathrm{Y}^{*}\right)$ underlying the ordered outcome has a heteroskedastic error term, even conditional on all independent variables. There are two possible sources of heteroskedasticity. The first is correlation between the errors in match outcomes within a team. This makes intuitive sense, since each team may have not only an individual intercept (i.e. team fixed effect) but an individual variance in outcomes (i.e., some teams may play more consistently than others). I test for this by running an ordered logit model with the full set of independent variables (X) from Section 3 , assuming an exponentially distributed variance. I omit team fixed-effects, but include indicators for 14 of the 15 clubs in the variance equation (Appendix A - Table A1). ${ }^{13}$ Fixed effects are omitted from the linear index for $\mathrm{Y}^{*}$ since within each season, each team has only 17 observations. This would leave only 8.5 observations to identify the two parameters for each club: dangerously close to the limit on the number needed to avoid inconsistent estimates. I assume that if the errors are uncorrelated within clubs without fixed effects, then they will not be correlated when the fixed effects are included back into the model.

Running this model I find that only 1 of 14 coefficients in the variance equation is significant at the $10 \%$ level, and I fail to reject the null hypothesis of joint insignificance $(p=0.65)$. Therefore, I conclude that there is no significant correlation among matches played by a single team, conditional on all other independent variables.

The second potential source of heteroskedasticity is the standard interpretation that the conditional variance of $Y^{*}$ is a function of one or more of the variables in X. A theoretical case for heteroskedasticity can be made for many of the variables in X. For instance, visiting teams may play more inconsistently due to

\footnotetext{
${ }^{13}$ All ordered analyses use the OGLM user-written command, attributable to Williams (2010).
}

travel fatigue, crowd effects, or the potential effect of AG. To test for general heteroskedasticity, I run an ordered logit model with the full set of independent variables from Section 3 (including team and year fixed effects). The exponentially distributed variance equation includes linear (but not quadratic) terms for the difference in points between the two teams and match attendance, as well as the distance variable, indicators for the 6 time zones, and an indicator for AG. Results are presented in Appendix A, Table A2. The distance variable, as well as 3 of the 6 time zone indicators, is significantly correlated with the variance of the latent index. Attendance and AG both have a $p$-value less than 0.20 in the variance equation, and the full vector of controls in the variance equation are jointly significant $(p=0.09)$. Therefore, I reject the null hypothesis of homoscedasticity and keep these controls for heteroskedasticity in the final model. ${ }^{14}$ Results of the final model are presented in Table 3. Estimates for all variables are reported as raw coefficients. I also report marginal effects for AG, which express the percentage point change in the probability of winning, drawing, or losing attributable to AG.

Estimates of match-level outcomes suggest that AG is uncorrelated with the outcome of an individual match, regardless of specification. A simple model with no control variables predicts that AG reduces the probability of winning for the visitor by approximately 1.8 percentage points, while increasing the probability of a loss by 2.6 percentage points. In the preferred model with visitor fixed effects and modeled heteroskedasticity, the probability of victory for the visitor increases by roughly 2.6 percentage points, while the probability of losing also increases by 1.3 percentage points. The z-statistic for the marginal effect of AG on the probability of winning, drawing, or losing, is never larger than 1.35 (for a 3.9 percentage point decrease in the probability of drawing) although all other marginal effects in all specifications have a z-score of 1 or less. The results of the preferred model are all inconsistent with the theoretical model, although they are not statistically significant.

\footnotetext{
${ }^{14}$ It is possible that the test of proportional odds is invalidated due to the presence of heteroskedasticity in the latent index. However, as seen in Table 3 the inclusion of controls for heteroskedasticity does not have a substantive impact on coefficient estimates. Estimating a generalized Hausmann test, I fail to reject the null hypothesis that the coefficient estimates are equal between the homoscedastic and heteroskedastic models $(p=1.00)$. This suggests that the proportional odds assumption holds even in the presence of heteroskedasticity.
} 
Table 3

Ordered logistic regression model of match outcomes for visiting team

\begin{tabular}{|c|c|c|c|c|}
\hline & 1 & 2 & 3 & 4 \\
\hline \multicolumn{5}{|l|}{ Marginal Effects of Artificial Grass } \\
\hline Change in Probability of Win & $-0.018(0.024)$ & $-0.008(0.027)$ & $0.026(0.032)$ & $0.026(0.032)$ \\
\hline Change in Probability of Draw & $-0.007(0.010)$ & $-0.003(0.010)$ & $-0.030(0.030)$ & $-0.039(0.029)$ \\
\hline Change in Probability of Loss & $0.026(0.033)$ & $0.010(0.037)$ & $0.033(0.036)$ & $0.013(0.036)$ \\
\hline \multicolumn{5}{|l|}{ Coefficient Estimates } \\
\hline Artificial Grass & $-0.103(0.134)$ & $-0.044(0.160)$ & $-0.014(0.229)$ & $-0.080(0.243)$ \\
\hline Difference in Points & & $0.073^{* *}(0.037)$ & $0.079(0.026)$ & $0.103 *(0.057)$ \\
\hline Difference in Points Squared & & $-0.006(0.004)$ & $-0.001(0.001)$ & $-0.001(0.001)$ \\
\hline Attendance (1000) & & $-0.099 * *(0.046)$ & $-0.123^{*}(0.071)$ & $-0.120 *(0.070)$ \\
\hline Attendance Squared (1 million) & & $0.002 *(0.001)$ & $0.002(0.001)$ & $0.001(0.001)$ \\
\hline Distance & & $-0.032 *(0.017)$ & $-0.042^{*}(0.024)$ & $-0.038(0.027)$ \\
\hline East 1 time zone & & $-0.040(0.019)$ & $-0.087(0.263)$ & $-0.092(0.277)$ \\
\hline East 2 time zones & & $0.272(0.369)$ & $0.303(0.519)$ & $0.351(0.528)$ \\
\hline East 3 time zones & & $0.611(0.355)$ & $1.002 *(0.556)$ & $0.823(0.555)$ \\
\hline West 1 time zone & & $0.179(0.242)$ & $0.327(0.329)$ & $0.222(0.354)$ \\
\hline West 2 time zones & & $0.075(0.271)$ & $0.123(0.416)$ & $-0.093(0.429)$ \\
\hline West 3 time zones & & $0.595(0.436)$ & $0.762(0.617)$ & $0.668(0.671)$ \\
\hline$\mu_{1}$ & $-0.057(0.073)$ & $-0.208(0.841)$ & $-0.411(1.104)$ & $-0.245(1.24)$ \\
\hline$\mu_{2}$ & $1.15^{* * *}(0.082)$ & $1.073(0.841)$ & $1.389(1.075)$ & $1.628(1.231)$ \\
\hline Heteroskedasticity controls & No & No & Yes & Yes \\
\hline Year Fixed Effects & No & Yes & Yes & Yes \\
\hline Team Fixed Effects & No & Yes & No & Yes \\
\hline
\end{tabular}

${ }^{*} p<0.1,{ }^{* *} p<0.05,{ }^{* * *} p<0.01$. Statistical significance determined by z-test. Standard errors in parentheses. $\mathrm{N}=1003$. Marginal effects indicate percentage point change in probability of a given match outcome for the visiting team attributable to artificial grass. $\mu_{1}$ indicates the cutoff between losing and drawing on the latent index $\mathrm{Y}^{*} . \mu_{2}$ indicates the cutoff between drawing and winning on the latent index $\mathrm{Y}^{*}$. Year fixed effects refer to dummy variables for each season from 2011-2014 with 2011 as the omitted year. Team fixed effects refer to dummy variables for each visiting club in the sample with Chivas USA as the omitted club. Heteroskedasticity controls indicate that the variance of the latent index is modeled as an exponential function of a linear index containing the following variables: artificial grass, difference in points, attendance, distance, and all 6 time zone indicators.

Table 4

Restricted generalized poisson regression model of goals scored and goals conceded by visiting team

\begin{tabular}{|c|c|c|c|c|c|c|}
\hline & \multicolumn{3}{|c|}{ Goals Scored By Visitor } & \multicolumn{3}{|c|}{ Goals Conceded by Visitor } \\
\hline & 1 & 2 & 3 & 4 & 5 & 6 \\
\hline $\begin{array}{l}\text { Marginal Effect of Artificial } \\
\text { Grass on Goals }\end{array}$ & $-0.012(0.022)$ & $-0.007(0.023)$ & $-0.012(0.023)$ & $0.018(0.024)$ & $0.014(0.027)$ & $0.015(0.027)$ \\
\hline \multicolumn{7}{|l|}{ Coefficient Estimates } \\
\hline Artificial Grass & $-0.040(0.068)$ & $-0.023(0.077)$ & $-0.41(0.078)$ & $0.041(0.053)$ & $0.035(0.062)$ & $0.032(0.061)$ \\
\hline Difference in Points & & $0.026(0.017)$ & $0.025(0.018)$ & & $-0.008(0.012)$ & $-0.011(0.011)$ \\
\hline $\begin{array}{l}\text { Difference in } \\
\text { Points Squared }\end{array}$ & & $-0.000(0.000)$ & $-0.000(0.000)$ & & $-0.000(0.000)$ & $-0.000(0.000)$ \\
\hline Attendance (1000) & & $-0.045^{* *}(0.021)$ & $-0.045^{* *}(0.021)$ & & $0.055^{* * *}(0.018)$ & $0.055^{* * * *}(0.018)$ \\
\hline $\begin{array}{l}\text { Attendance Squared } \\
\text { (1 million) }\end{array}$ & & $0.001 *(0.000)$ & $0.001^{*}(0.000)$ & & $-0.001^{* * *}(0.000)$ & $-0.001^{\text {**** }}(0.000)$ \\
\hline Distance & & $-0.003(0.008)$ & $-0.003(0.008)$ & & $0.011^{*}(0.006)$ & $0.012^{* *}(0.006)$ \\
\hline East 1 time zone & & $-0.025(0.088)$ & $-0.050(0.094)$ & & $-0.006(0.073)$ & $-0.015(0.070)$ \\
\hline East 2 time zones & & $0.184(0.170)$ & $0.172(0.172)$ & & $0.187(0.128)$ & $0.156(0.122)$ \\
\hline East 3 time zones & & $0.186(0.166)$ & $0.067(0.170)$ & & $-0.146(0.138)$ & $-0.150(0.137)$ \\
\hline West 2 time zones & & $0.040(0.125)$ & $-0.040(0.131)$ & & $-0.036(0.102)$ & $-0.097(0.097)$ \\
\hline West 3 time zones & & $0.190(0.201)$ & $0.130(0.210)$ & & $-0.065(0.162)$ & $-0.118(0.155)$ \\
\hline Constant & $0.103^{* * *}(0.035)$ & $0.130(0.365)$ & $0.211(0.404)$ & $0.447^{* * *}(0.029)$ & $-0.054(0.313)$ & $-0.072(0.283)$ \\
\hline Dispersion Parameter & $0.001(0.020)$ & $-0.018(0.019)$ & $-0.017(0.019)$ & $-0.031^{* *} 0.013$ & $-0.052^{* * *}(0.012)$ & $-0.046^{* * * *}(0.012)$ \\
\hline Year Fixed Effects & No & Yes & Yes & No & Yes & Yes \\
\hline Team Fixed Effects & No & No & Yes & No & No & Yes \\
\hline
\end{tabular}

${ }^{*} p<0.1,{ }^{* *} p<0.05,{ }^{* * *} p<0.01$. Statistical significance determined by z-test. Standard errors in parentheses. $\mathrm{N}=1003$. Marginal effects indicate change in expected number of goals scored or conceded by the visiting team attributable to artificial grass. Year fixed effects refer to dummy variables for each season from 2011-2014 with 2011 as the omitted year. Team fixed effects refer to dummy variables for each visiting club in the sample with Chivas USA as the omitted team. 
The remainder of the results support the validity of the model. Consistent with expectations, the difference between visitors' points earned at home and the home team's points earned on the road is correlated with better match outcomes (i.e. higher probability of tying or winning) for the visitor, although the quadratic term is insignificant, suggesting the relationship may be linear. Consistent with the literature, higher home attendance disadvantages the visiting team. The quadratic term is of the opposite sign, suggesting there are decreasing returns to the home team as attendance increases. Distance traveled is also negatively correlated with match outcomes for the visiting team, consistent with the literature. Conditional on distance, changes in time zones are positively correlated with match outcomes (although only one of the coefficients is significant). This is a counter-intuitive result, which holds even in a model that omits the distance variable. This may suggest a discrepancy in skill between teams on the west and east coasts that is not captured by the quadratic proxy for skill difference.

\subsection{Goals scored and conceded}

As for goals occurring during a match, I assume that both $\mathrm{G}_{\mathrm{V}}$ (goals scored) and $\mathrm{G}_{\mathrm{H}}$ (goals conceded) follow a count distribution (since the outcomes are non-negative and discrete). I also assume the two goal outcomes have an exponential conditional mean: a standard assumption for parametric count models (which also upholds the continuity of $\mathrm{E}\left[\mathrm{G}_{\mathrm{H}}\right]$ and $\mathrm{E}\left[\mathrm{G}_{\mathrm{V}}\right]$ asserted in Section 2). These two assumptions are shared by the following studies modeling goals scored and/or conceded in soccer matches: Goddard (2005); Everson and Goldsmith-Pinkham (2008); Chumacero (2007); Baio and Blangiardo (2010); and Dixon and Coles (1997). This suggests the use of a Poisson model. However, the Poisson model makes the potentially restrictive assumption that the mean number of goals scored, after controlling for $\mathrm{X}$, is equal to the variance of goals scored. That is, $E\left[G_{V} \mid X\right]=V\left[G_{V} \mid X\right]$ (and likewise for $G_{H}$ : the so-called "equidispersion" assumption.) Although the model remains consistent if this assumption is violated, the model will be inefficient relative to a correctly specified distribution, inflating the size of the standard errors.

I utilize a test proposed by Cameron and Trivedi (2005, pp. 671) to determine the validity of this assumption. This test is described in Appendix B. I fail to reject the null hypothesis of equidispersion for goals scored, yielding a t-statistic of -0.93 . However,
I do reject the null hypothesis for goals conceded with a t-statistic of -4.43 , indicating data that are conditionally underdispersed (i.e., the variance is less than the mean after controlling for X). I therefore estimate the model using the restricted generalized Poisson (RGP) (Famoye, 1993), which allows for limited underdispersion in the dependent variable. ${ }^{15}$ There is no need to control for potential heteroskedasticity in the model since parametric count data models incorporate the effect of each variable on the variance of the outcome. Estimates from RGP models for goals scored and goals conceded are presented in Table 4. Estimates for all variables are reported as raw coefficients. I also report marginal effects for AG, which express the change in expected goals scored and conceded attributable to AG.

AG is negatively correlated with goals scored by the visiting team, consistent with the theoretical prediction. However, the effect is about 0.01 goals per game, and is statistically insignificant. AG is positively correlated with goals conceded by the visiting team (roughly 0.02 goals per game), although this result is also substantively and statistically insignificant. The negative and statistically significantestimate for the dispersion parameter in the model for goals conceded is consistent with the results of the test for equidispersion, and vindicates the use of the restricted generalized Poisson model.

Although AG is uncorrelated with match outcomes, it is conceivable that both the home and visiting teams are equally disadvantaged by AG such that overall goal-scoring falls and the results stay the same. However, the lack of change in both goals scored and goals conceded suggest that this is not the case. As with the ordered model, the remainder of the results support the validity of the models for goals scored and goals conceded, as the coefficients generally have the expected signs (the time-zone coefficients remain an exception - albeit an insignificant one).

\section{Robustness checks and discussion}

The results presented above rest on several key assumptions. The first and most important is that teams with AG do not face a disadvantage playing on

\footnotetext{
${ }^{15}$ The proportion of matches with zero, one, or two goals scored by the visiting team is $0.33,0.37$, and 0.20 , respectively. The proportion of matches with zero, one, or two goals conceded by the visiting team is $0.19,0.33$, and 0.28 . Therefore, I conclude that the data do not suggest an "excess" of zeros that needs to be modeled by a separate process.
} 
their own field. I argue that this is the case because players who regularly perform on AG should not have their play affected to the same degree by AG. Moreover, if AG really does increase player fatigue, or change movement of the ball, then players should become accustomed to this over time. One way to look for this is to see if the alleged effects of playing on AG such as player fatigue and/or injury have a cumulative effect. If teams that play on AG suffer from greater rates of injury or fatigue, then we should see their performance drop as the season progresses relative to teams playing on grass home surfaces. To test for this, I create season-level measures of the difference in total points earned in the first 17 games of the season, and points earned in the second 17 games of the season for each team $(n=75)$. I then run a simple OLS regression with year fixed effects and an indicator for AG to see if teams with AG earn fewer points in the second half of the season relative to the first half, compared to teams with real grass home fields.

On average, teams only perform a half point worse in the second half of the season than the first half, although the distribution is large (maximum 23, minimum -18 , standard deviation 7.92). The four teams with AG are predicted to have an increased difference in performance between the first and second half of the season although the coefficient is miniscule ( 0.28 points - less than $1 / 25$ of a standard deviation) and not close to significant $(P=0.90)$. This test is not particularly rigorous, as it cannot account for potential differences in cumulative travel between teams or other potential confounders. However, it does serve as suggestive evidence that teams with AG are not disadvantaged by their own surface.

Another key assumption of the model is that the difference between the visiting team's home points and the home team's away points is a valid proxy for the underlying differences in talent between the two teams, even though points earned on the road are not a monotonic transformation of points earned at home. As mentioned previously, so long as the difference between points earned at home and points earned on the road is not a function of AG, then this is simply measurement error that will attenuate the coefficient for the difference in points. To test this, I create seasonlevel measures of the difference between points earned at home and points earned on the road. The average season played has a difference of 16.8 points between home and road matches (maximum 28, minimum -2, standard deviation 6.01). Regressing the difference in points on AG with year fixed effects using OLS, the model indicates that teams with AG have an increased difference of 0.37 points between home and away matches $(P=0.83)$. This suggests that possible measurement error in the difference in talent between teams is not a function of AG and is not biasing the results.

There are several other shortcomings of the model. First, Nichols (2012) suggests that differences in temperature may affect the outcome of NFL matches, and it is reasonable to assume the same about soccer. Although I do not control for temperature differences in the week of the match, I contend that this should not adversely affect the results. The MLS season runs from mid-March through mid-October. The Pacific Northwest is a temperate climate, and so three of the four teams with AG should have fairly stable, albeit cooler than average, temperatures. Teams used to playing in 80 or 90 degree temperatures during the summer should not be physically disadvantaged playing in 60 or 70 degree temperatures (unlike NFL teams, which may transition from 80 degree home temperatures to 20 degree road temperatures in the middle of winter). While New England may be unusually cold near the end of the season, the average temperature in Boston in October ranges from lows in the mid-40's to highs in the mid-60's (Walsh, 2015). I contend that this is not sufficiently cold to disadvantage a visiting team and potentially bias the AG coefficient. Moreover, if New England gained a cold-weather advantage, it would bias the coefficient on AG downward. Given that the actual estimated coefficient is zero, if the estimate was biased, it would indicate that the "true" effect of AG was positive - that is, that AG disadvantaged the home teams playing on them. This result is inconsistent with the conceptual model, and suggests that the results are not biased.

Of greater potential concern is that teams with AG playing surfaces may themselves be disadvantaged by heat when playing on the road. This would decrease the number of points they earned on the road relative to their true talent level, which would upward bias the estimated difference in talent between them and their opponents. The consequence would be an increase in $\mathrm{Y}^{*}$ for the visitor compared to the $\mathrm{Y}^{*}$ that would be predicted by the "true" difference in talent between the two teams. In order for $Y^{*}$ to be decreased back to its "true" level (i.e., the actual outcome of the match), the coefficient on AG will have to be smaller. Therefore, the net effect of the measurement error would be a downward bias on the AG coefficient. Again, given that the estimated effect is insignificantly different from zero, the presence of such a bias would 
indicate that the true, unbiased effect of AG is to disadvantage the home team, which is inconsistent with the conceptual model.

Another potential weather confounder is precipitation: something for which the Pacific Northwest is notorious, and which may disadvantage visiting teams not as used to playing in wet conditions. To test for the possible impact of weather I run season-level OLS regressions of points earned at home on average annual precipitation and also on average number of days with precipitation. Weather data are obtained from Current Results: Weather and Science Facts (2015), which averages data from 1981-2010. Teams earn an average of 29.5 points at home per season (maximum 41, minimum 12, standard deviation 6.83). Each additional inch of precipitation is associated with a -0.016 reduction in expected points earned at home per season $(P=0.79)$, while each additional day of precipitation is associated with earning 0.004 fewer points at home per season $(P=0.86)$. This suggests that precipitation is, on average, uncorrelated with match outcomes, and the correlation between precipitation and AG should not bias the estimated outcomes. Combined, these additional results suggest that the results presented in Section 4 are valid.

\section{Conclusion}

In this study I consider the relationship between artificial grass playing surfaces and the competitive balance in MLS. I estimate an ordered logit model for the loss/draw/win sequence, as well as a restricted generalized Poisson model for goals scored by visiting teams and goals conceded by visiting teams. Although there are several player perceptions that suggest that teams used to playing on grass would be disadvantaged playing on AG, I fail to find any evidence to support this hypothesis across the four seasons spanning 2011 to 2014 . The remaining results are generally consistent with previous literature, suggesting the models are valid. Moreover, the failure to reject the null hypothesis that $\mathrm{AG}$ is uncorrelated with match outcomes or goals cannot be attributed to lack of power as the coefficients for AG are within one decimal place of zero. ${ }^{16}$ There may be much more to the entertainment value of a game than goals scored

\footnotetext{
${ }^{16}$ In 2010 only two teams had AG surfaces, while in 2009 and 2008 there were three. This suggests that adding additional years of data would only slightly increase power. Given the small magnitude of the predicted effect, it is unlikely that additional data would yield a different conclusion.
}

or points earned, and so I cannot conclude that the fan experience is unchanged by AG. However, the results suggest that, on average, clubs that play on AG do not earn an unfair advantage over opponents who play on natural grass surfaces, nor are the total number of goals scored different than if the game were played on grass.

\section{Acknowledgments}

The author would like to thank Ian Breunig and Allison Marier for their helpful comments. The author also acknowledges the two anonymous reviewers for investing their time to help make this paper as high-quality as possible. Lastly, the author would like to thank Matthew Rabbitt, whose gift provided the original motivation for this paper

\section{References}

Allison, P.D. \& Waterman, R.P. (2002). Fixed effects negative binomial regression models. Sociological Methodology, 32, 247-265.

Andersson, H., Ekblom, B. \& Krustrup, P. (2008). Elite football on artificial turf versus natural grass: Movement patterns, technical standards, and player impressions. Journal of Sports Sciences, 26(2), 113-122.

Arabzad, S.M., Araghi, M.E.T. \& Soheil, S.N. (2014). Football match results prediction using artificial neural networks; the case of Iran Pro League. International Journal of Applied Research on Industrial Engineering, 1(3), 159-179.

Baio, G. \& Blangiardo, M. (2010). Bayesian hierarchical model for the prediction of footballresults. Journal of Applied Statistics, $37(2), 253-264$

Barnett, V. \& Hilditch, S. (1993). The Effect of an artificial pitch surface on home teamperformance in football (soccer). Journal of the Royal Statistical Society: Series A(Statistics in Society), 156(1), 39-50

Bell, J. Apr. 28, (2009). So, artificial grass is a good thing? Goal: The New York Times SoccerBlog. New York Times. Available from: http://goal.blogs.nytimes.com/2009/04/28/and-this-isa-good-thing/?_r=0

Brant, R. (1990). Assessing proportionality in the proportional odds model for ordinal logistic regression. Biometrics, 46(4), 1171-1178.

Buis, M.L. (2013). Oparallel: Stata module providing postestimation command for testing the parallel regression assumption

Cameron, C \& P. Trivedi. (2005). "Microeconometrics: Methods and Applications" Cambridge University Press. 671.

Chumacero, R. (2007). Altitude or hot air? MPRA Working Paper No. 15178.

Clarke, S. \& Norman, J. (1995). Home ground advantage of individual clubs in English soccer. The Statistician, 44(4), 509-521. 
Corr, C. June 8, (2009). World Cup qualifier special, Part VIII: Bob Bradley wants FIFA to ban artificial turf. Available from: http://www.examiner.com/article/world-cup-qualifierspecial-part-viii-bob-bradley-wants-fifa-to-ban-artificial-turf

Current Results: Weather and Science Facts February 10, (2015). Average Annual Precipitationby City. Available from: http://www.currentresults.com/Weather/US/average-annualrecipitation-by-city.php http://www.currentresults.com/ Weather/Canada/Cities/precipitation-annual- average.php

Davidson, N. Oct. 27, (2014). "MLS players sympathize with women over use of World Cupartificial turf." 1310 News. Available from: http://www.1310news.com/2014/10/27/mlsplayers-sympathize-with-women-over-use-of-world-cupartificial-turf/

Davis, S. Sep. 14, (2013). Bruce Arena calls artificial turf in MLS "disasters" NBC Sports. NBC. Available from: http://prosoccertalk.nbcsports.com/2013/09/14/brucearena-calls- artificial-turf-in-mls-disasters/

Dixon, M. \& Coles, S. (1997). Modelling association football scores and inefficiencies in the football betting market. Applied Statistics, 46(2), 265-280.

Dewenter, R. \& Namini, J.E. (2013). How to make soccer more attractive? Rewards for avictory, the teams' offensiveness, and the home bias. Journal of Sports Economics, 14(1), 65-86.

DistanceFromTo. August 5, (2014). "Distance between cities places on maps." Available from: http://www. distancefromto.net/

Everson, P. \& Goldsmith-Pinkham, P. (2008). Composite Poisson models for goal scoring. Journal of Quantitative Analysis in Sports, 4(2), 13.

Famoye, F. (1993). Restricted generalized Poisson regression model. Communications in Statistics-Theory and Methods, 22(5), 1335-1354.

Goddard, J. (2005). Regression models for forecasting goals and match results in associationfootball. International Journal of Forecasting, 21(2), 331-340.

Goddard, J. \& Asimakopoulos, I. (2004). Forecasting football results and the efficiency of fixed-odds betting. Journal of Forecasting, 23(1), 51-66.

Goumas, C. (2014a). Home advantage in Australian soccer. Journal of Science and Medicine in Sport, 17(1), 119-123.

Goumas, C. (2014b). Tyranny of distance: Home advantage and travel in international clubfootball. International Journal of Performance Analysis in Sports, 14(1), 1-13.

Graham, I., \& Stott, H. (2008). Predicting bookmaker odds and efficiency for UK football. Applied Economics, 40(1), 99-109.
Gwynne, P. Sep 23, (2014). Elite women soccer players challenge plan to play next worldcup on artificial turf. Inside Science. Available from: http://www.insidescience.org/content/elitewomen-soccer-players-challenge-plan-play-next-world-cupartificial-turf/2071

Heckman, J. (1981). The incidental parameters problem and the problem of initial conditions inestimating a discrete time-discrete data stochastic process. Structural Analysis ofDiscrete Data and Econometric Applications. Edited by C.D. Manski and D.L.McFadden. Cambridge: MIT press.

Koreen, E. August 4, (2007). Toronto's FieldTurf may keep Beckham off the pitch. Canwest News Service Available from: http://www.canada.com/story_print.html?id=9c018f61-3951 -474f-b3ec-1ed019d4688f\&sponsor=MajorLeagueSoccer, August 1, 2014 Available from: http://www.mlssoccer.com/

Mccann, M. Oct 31, (2014). Players' anti-turf lawsuit for Women's World Cup not a clear-cutwin. Sports Illustrated. Available from: http://www.si.com/soccer/planet-futbol/2014/10/31/ womens-world-cup-artificial-turf-lawsuit-analysis-wwc

Nichols, M. (2012). The Impact of visiting team travel on game outcome and biases in NFL betting markets. Journal of Sports Economics, 15(1), 78-96.

Oberhofer, H., Philippovich, T. \& Winner, H. (2010). Distance matters in away games: Evidencefrom the German football league. Journal of Economic Psychology, 31, 200-211.

Pazino, F. June 5, (2014). “New York Red Bulls' Jamison Olave Likely out vs. New EnglandRevolution due to turf playing surface." Major League Soccer. Available from: http://www.mlssoccer.com/news/article/2014/06/05/newyork-red-bulls-jamison-olave-likely-out-vs-new-englandrevolution-due-turf

Ponzo, M. \& Scoppa, V. (2014). Does the home advantage depend on crowd support? Evidence from same-stadium derbies. IZA Discussion Paper No. 8105.

Poulous, C., Galluci, J. Gage, W.H., Baker, J., Buitrago, S. \& Macpherson, A.K. (2014). The perceptions of professional soccer players on the risk of injury from competition and training on natural grass and 3rd generation artificial turf." BMC Sports Science, Medicine, and Rehabilitation, 6(11).

Walsh, K. February 10, (2015). Typical weather in Boston in October. Travel Tips by Demand Media. USA Today. Available from: http://traveltips.usatoday.com/typical-weather-bostonoctober-102091.html

Williams, R. (2010). Fitting heterogenous choice models with OGLM Stata Journal, 10(4), 540-567. 


\section{Appendix A - Heteroskedasticity tests for ordered logit model}

Table A1

Test for conditional correlation of team errors across seasons

\begin{tabular}{lc}
\hline $\begin{array}{l}\text { Team dummy } \\
\text { variables }\end{array}$ & $\begin{array}{c}\text { Coefficient in } \\
\text { linear index for } \\
\text { exponential variance }\end{array}$ \\
\hline Team 1 & $0.033(0.032)$ \\
Team 2 & $0.038(0.033)$ \\
Team 3 & $0.242(0.356)$ \\
Team 4 & $0.435(0.342)$ \\
Team 5 & $0.150(0.338)$ \\
Team 6 & $0.069(0.320)$ \\
Team 7 & $-0.015(0.320)$ \\
Team 8 & $0.098(0.335)$ \\
Team 9 & $-0.025(0.310)$ \\
Team 10 & $0.207(0.325)$ \\
Team 11 & $0.346(0.331)$ \\
Team 12 & $0.038(0.330)$ \\
Team 13 & $0.576^{*}(0.330)$ \\
Team 14 & $-0.139(0.322)$ \\
\hline
\end{tabular}

Joint significance: $P=0.65 .{ }^{*} p<0.1$. Statistical significance determined by $\mathrm{z}$-test. Linear index for $\mathrm{Y}^{*}$ contains controls for $\mathrm{AG}$, difference in points, squared difference in points, mean attendance, attendance squared, distance traveled, indicators for time zone changes, and season fixed effects. Standard Errors in parentheses.

Table A2

Test for Heteroskedastic errors

\begin{tabular}{lc}
\hline Variable & $\begin{array}{c}\text { Coefficient in } \\
\text { linear index for } \\
\text { exponential variance }\end{array}$ \\
\hline Artificial Grass & $0.191(0.137)$ \\
Difference in Points & $0.005(0.007)$ \\
Mean Attendance & $0.014(0.010)$ \\
Distance & $-0.036^{* *}(0.014)$ \\
East 1 time zone & $0.324^{* *}(0.164)$ \\
East 2 time zones & $0.418(0.333)$ \\
East 3 time zones & $0.333(0.309)$ \\
West 1 time zone & $0.222(0.200)$ \\
West 2 time zones & $0.668^{* * *}(0.229)$ \\
West 3 time zones & $0.617^{*}(0.350)$ \\
\hline
\end{tabular}

Joint significance: $P=0.09 .{ }^{*} p<0.1,{ }^{* *} p<0.05,{ }^{* * *} p<0.01$. Statistical significance of individual variables determined by z-test. Joint significance determined by likelihood ratio test. Linear index for $\mathrm{Y}^{*}$ contains controls for AG, difference in points, squared difference in points, mean attendance, attendance squared, distance traveled, indicators for time zone changes, and season fixed effects. Standard Errors in parentheses.

\section{Appendix B - Dispersion test}

The test is as follows:

- Estimate a standard Poisson model.

- Estimate $\hat{\mu}=\exp (X \hat{\beta})$, where $\hat{\beta}$ is the vector of coefficient estimates from the Poisson model, and $\mathrm{X}$ is the vector of independent variables.

- Estimate the following equation (without a constant) using OLS.

$$
\frac{(Y-\hat{\mu})^{2}-Y}{\hat{\mu}}=\alpha \frac{\hat{\mu}^{2}}{\hat{\mu}}+u
$$

where $Y$ is the count outcome of interest.

- If $\alpha$ is significantly greater than 0 , the data are overdispersed. If $\alpha$ is significantly less than 0 , the data are underdispersed. 
Appendix C - Results of alternate specifications for difference in skill between two teams

Table C1

Ordered logistic regression model of match outcomes for visiting team by specification of difference in skill

\begin{tabular}{|c|c|c|c|}
\hline & $\begin{array}{l}\text { Points from } \\
\text { entire season }\end{array}$ & $\begin{array}{l}\text { Win-Ratio from } \\
\text { entire season }\end{array}$ & $\begin{array}{l}\text { Points in } \\
\text { most recent } \\
4 \text { matches }\end{array}$ \\
\hline \multicolumn{4}{|l|}{ Marginal Effects of Artificial Grass } \\
\hline Change in Probability of Win & $0.026(0.032)$ & $0.025(0.032)$ & $0.005(0.036)$ \\
\hline Change in Probability of Draw & $-0.039(0.029)$ & $-0.037(0.029)$ & $-0.031(0.030)$ \\
\hline Change in Probability of Loss & $0.013(0.036)$ & $0.012(0.036)$ & $0.025(0.034)$ \\
\hline \multicolumn{4}{|l|}{ Coefficient Estimates } \\
\hline Artificial Grass & $-0.080(0.243)$ & $-0.068(0.236)$ & $-0.022(0.181)$ \\
\hline Difference in Skill & $0.103 *(0.057)$ & $5.431^{*}(2.89)$ & $-0.041(0.085)$ \\
\hline Difference in Skill Squared & $-0.001(0.001)$ & $-2.241(2.19)$ & $0.004(0.004)$ \\
\hline Attendance $(1000)$ & $-0.120 *(0.070)$ & $-0.114 *(0.069)$ & $-0.094 *(0.056)$ \\
\hline Attendance Squared (1 million) & $0.001(0.001)$ & $0.001(0.001)$ & $0.001(0.001)$ \\
\hline Distance & $-0.038(0.027)$ & $-0.037(0.027)$ & $-0.023(0.020)$ \\
\hline East 1 time zone & $-0.092(0.277)$ & $-0.093(0.273)$ & $-0.089(0.219)$ \\
\hline East 2 time zones & $0.351(0.528)$ & $0.340(0.515)$ & $0.111(0.414)$ \\
\hline East 3 time zones & $0.823(0.555)$ & $0.814(0.557)$ & $0.549(0.421)$ \\
\hline West 1 time zone & $0.222(0.354)$ & $0.208(0.345)$ & $0.072(0.276)$ \\
\hline West 2 time zones & $-0.093(0.429)$ & $-0.099(0.419)$ & $-0.172(0.326)$ \\
\hline West 3 time zones & $0.668(0.671)$ & $0.553(0.657)$ & $0.471(0.490)$ \\
\hline$\mu_{1}$ & $-0.245(1.24)$ & $-0.025(1.239)$ & $-1.957(1.049)$ \\
\hline$\mu_{2}$ & $1.628(1.231)$ & $1.810(1.253)$ & $-0.526(0.844)$ \\
\hline Log Likelihood [Psedo R2] & $-998.80[0.046]$ & $-999.22[0.045]$ & $-1009.15[0.036]$ \\
\hline
\end{tabular}

${ }^{*} p<0.1,{ }^{* *} p<0.05,{ }^{* * *} p<0.01$. Standard errors in parentheses. $\mathrm{N}=1003 . \mu_{1}$ indicates the cutoff between losing and drawing on the latent index $\mathrm{Y}^{*} . \mu_{2}$ indicates the cutoff between drawing and winning on the latent index $\mathrm{Y}^{*}$. Marginal effects indicate percentage point change in probability of a given match outcome for the visiting team attributable to artificial grass. All specifications include heteroscedasticity controls, as well as year and team fixed effects. Year fixed effects refer to dummy variables for each season from 2011-2014 with 2011 as the omitted year. Team fixed effects refer to dummy variables for each visiting club in the sample with Chivas USA as the omitted club. Heteroskedasticity controls indicate that the variance of the latent index is modeled as an exponential function of a linear index containing the following variables: artificial grass, difference in skill, attendance, distance, and all 6 time zone indicators. For each of the three specifications of the difference in skill, the specific skill measure reported in that column was also used as a control variable for heteroscedasticity. 


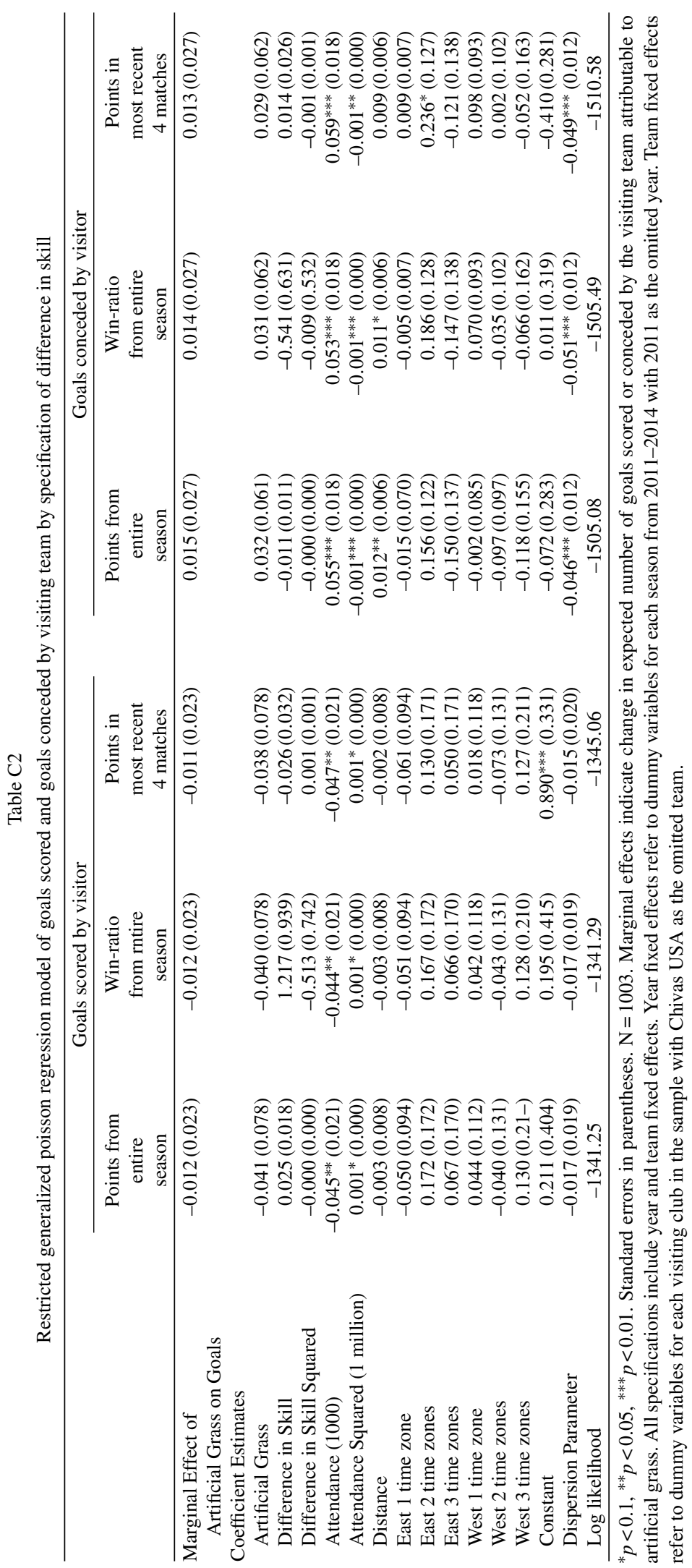

